Document downloaded from:

http://hdl.handle.net/10251/46242

This paper must be cited as:

Ballester-Bolinches, A.; Beidleman, J.; Heineken, H.; Pedraza Aguilera, MC. (2013).

Prefactorized subgroups in pairwise mutually permutable products. Annali di Matematica Pura ed Applicata. 192(6):1043-1057. doi:10.1007/s10231-012-0257-y.

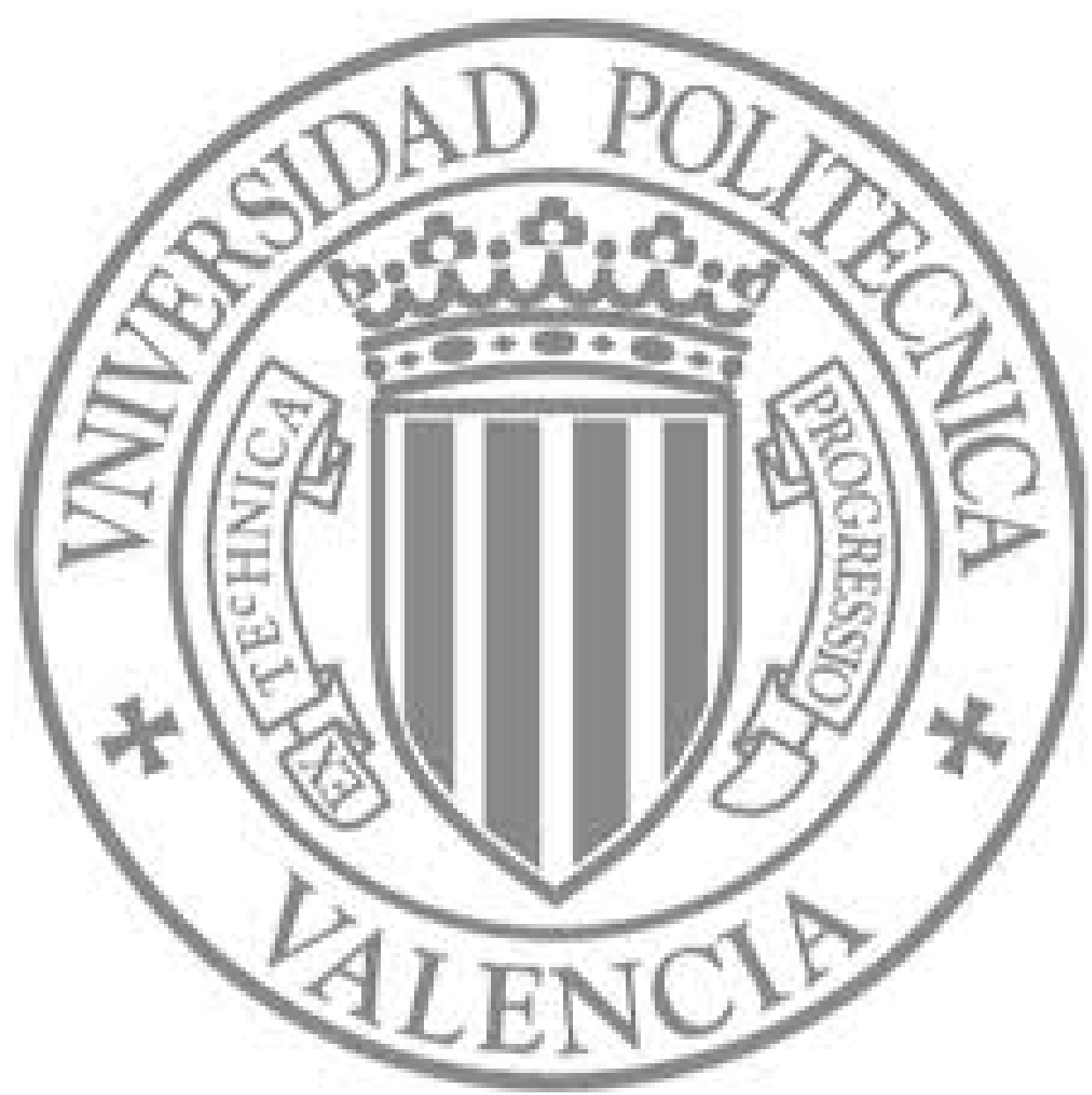

The final publication is available at

http://link.springer.com/article/10.1007/s10231-012-0257-y

Copyright Springer Verlag 


\title{
Prefactorized subgroups in pairwise mutually permutable products.
}

\author{
A. Ballester-Bolinches, J.C. Beidleman, H. Heineken and M.C. Pedraza-Aguilera
}

\begin{abstract}
We continue here our study of pairwise mutually and pairwise totally permutable products. We are looking for subgroups of the product in which the given factorization induces a factorization of the subgroup. In the case of soluble groups it is shown that a prefactorized Carter subgroup and a prefactorized system normalizer exists. A less stringent property have $\mathcal{F}$-residual, $\mathcal{F}$-projector and $\mathcal{F}$-normalizer for any saturated formation $\mathcal{F}$ including the supersoluble groups.

Mathematics Subject Classification (2000): 20D10, 20D20

Keywords: finite group, permutability, factorization, saturated formation
\end{abstract}

\section{Introduction and preliminaries}

All groups considered throughout this paper are finite.

A group $G$ is said to be the product of its subgroups $A$ and $B$ if $G=$ $A B$. Sometimes such a group is also called factorized by $A$ and $B$ or simply factorized. A subgroup $S$ of $G=A B$ is called prefactorized if $S=(S \cap$ $A)(S \cap B)$. We say that $S$ is factorized if whenever $s \in S$ and $s=a b$ with $a \in A$ and $b \in B$, then $a \in S$ (and $b \in S$ ). According to a result of Wielandt ([1, Lemma 1.1.1]), $S$ is factorized if and only if $S$ is prefactorized and $A \cap B \leq S$. In particular, every factorized subgroup of $G$ is prefactorized and every subgroup of $G$ containing $A$ or $B$ is factorized.

If $G=G_{1} G_{2} \ldots G_{k}$ is the pairwise permutable product of the subgroups $G_{1}, G_{2}, \ldots, G_{k}$, we say that a subgroup $S$ of $G$ is prefactorized with respect to the above factorization if $S=\left(S \cap G_{1}\right)\left(S \cap G_{2}\right) \ldots\left(S \cap G_{k}\right)$. 
A group $G=G_{1} G_{2} \ldots G_{k}$ is said to be the product of its pairwise mutually permutable subgroups $G_{1}, G_{2}, \ldots, G_{k}$, if $G_{i}$ and $G_{j}$ are mutually permutable subgroups of $G$, that is, $G_{i}$ permutes with every subgroup of $G_{j}$, and $G_{j}$ permutes with every subgroup of $G_{i}$ for all $i, j \in\{1,2, \ldots, k\}$. $G$ is said to be the pairwise totally permutable product of $G_{1}, G_{2}, \ldots, G_{k}$ if $G_{i}$ and $G_{j}$ are totally permutable subgroups of $G$, that is, every subgroup of $G_{i}$ permutes with every subgroup of $G_{j}$ for all $i \neq j$. These kind of products have been studied extensively with a lot of properties and results available (see $[2,3,4$, $5,6,7,8,9,10]$ and the papers cited therein).

We continue the investigation about certain subgroups of pairwise mutually and pairwise totally permutable products which began by the authors in [5] and [6]. The new results presented here are often related to saturated formations and to subgroups of the given product which are factorized or prefactorized.

In [5, Lemma 1(ii)], we proved that if $G=G_{1} G_{2} \ldots G_{k}$ is the pairwise mutually permutable product of $G_{1}, G_{2}, \ldots, G_{k}$ and $S$ is a subgroup of $G$, then $\left(S \cap G_{1}\right)\left(S \cap G_{2}\right) \ldots\left(S \cap G_{k}\right)$ is a subgroup of $G$ which is the pairwise mutually permutable product of its factors. Moreover if $S$ is a normal subgroup of $G$, then the above mentioned product is also a normal subgroup of $G$. We are concerned here with the case when this product coincides with the subgroup $S$ itself, that is, when $S$ is prefactorized with respect to $G=G_{1} G_{2} \ldots G_{k}$.

The following example shows where we should not try:

Example 1 Let $G=A \times B$ where $A=\langle u, v\rangle$ is a nonabelian group of order $p^{3}$ and $B=\langle x\rangle$ is a group of order $p$ with $p$ an odd prime. Then $A$ and $\langle u, v x\rangle$ are mutually permutable subgroups of $G$ and $G=A\langle u, v x\rangle$. We have $Z(G)=\langle[u, v], x\rangle$ and $Z(G) \cap A=Z(G) \cap\langle u, v x\rangle=\langle[u, v]\rangle \neq Z(G)$.

We begin with a result which allows us to extend the concept of factorized subgroup in the case of pairwise mutually permutable products.

Lemma 1. Let the group $G=G_{1} G_{2} \ldots G_{k}$ be the pairwise mutually permutable product of the subgroups $G_{1}, G_{2}, \ldots, G_{k}$. For a subgroup $S$ of $G$ the following conditions are equivalent.

(i) If $a_{i_{1}} a_{i_{2}} \ldots a_{i_{k}} \in S$, with $a_{i_{j}} \in G_{i_{j}}$, where $\left\{i_{1}, i_{2}, \ldots, i_{k}\right\}=\{1,2, \ldots, k\}$, 
then $a_{i_{j}}$ belongs to $S$ for all $i_{j} \in\{1,2, \ldots, k\}$.

(ii) $S=\left(S \cap G_{1}\right)\left(S \cap G_{2}\right) \ldots\left(S \cap G_{k}\right)$ and $G_{i} \cap \prod_{j \neq i} G_{j} \leq S$ for all $i=$ $1,2, \ldots, k$.

Proof (i) implies (ii) If $x=a_{1} a_{2} \ldots a_{k}$ is an element of $S$, with $a_{i} \in G_{i}$, then by the hypothesis $a_{i}$ belongs to $\left(S \cap G_{i}\right)$ for all $i=1,2, \ldots, k$. This shows that $S=\left(S \cap G_{1}\right)\left(S \cap G_{2}\right) \ldots\left(S \cap G_{k}\right)$. Moreover, if $x \in G_{i} \cap \prod_{j \neq i} G_{j}$ for some $i=1,2, \ldots, k$, then $x^{-1} \in \prod_{j \neq i} G_{j}$ and $x x^{-1}=1 \in S$. Thus by (i), $x$ lies in $S$.

(ii) implies (i) Let $x=a_{i_{1}} a_{i_{2}} \ldots a_{i_{k}}$ be an element of $S$ with $a_{i_{j}} \in G_{i_{j}}$ where $\left\{i_{1}, i_{2}, \ldots, i_{k}\right\}=\{1,2, \ldots, k\}$. Since $S=\left(S \cap G_{1}\right)\left(S \cap G_{2}\right) \ldots\left(S \cap G_{k}\right)$ and the factors are pairwise permutable, we have also that $x=b_{i_{1}} b_{i_{2}} \ldots b_{i_{k}}$ with $b_{i_{j}} \in$ $\left(S \cap G_{i_{j}}\right)$. Therefore $b_{i_{1}}^{-1} a_{i_{1}}=\left(b_{i_{2}} \ldots b_{i_{k}}\right)\left(a_{i_{2}} \ldots a_{i_{k}}\right)^{-1} \in G_{i_{1}} \cap G_{i_{2}} \ldots G_{i_{k}} \leq S$ by (ii) and so $a_{i_{1}} \in S$. Now we deduce that $a_{i_{2}} \ldots a_{i_{k}}$ belongs to $S$. Arguing as before, $a_{i_{2}} \ldots a_{i_{k}}=c_{i_{1}} c_{i_{2}} \ldots c_{i_{k}}=d_{i_{2}} d_{i_{1}} \ldots d_{i_{k}}$ with $c_{i_{j}} \in\left(S \cap G_{i_{j}}\right)$ and $d_{i_{j}} \in\left(S \cap G_{i_{j}}\right)$. Therefore $d_{i_{2}}^{-1} a_{i_{2}}$ belongs to $G_{i_{2}} \cap \prod_{j \neq i_{2}} G_{i_{j}}$ which is contained in $S$ by (ii). Consequently we obtain that $a_{i_{2}} \in S$ and similarly $a_{i_{3}}, \ldots, a_{i_{k}}$ belong to $S$.

A subgroup $S$ of a pairwise mutually permutable product $G=G_{1} G_{2} \ldots G_{k}$ is said to be factorized if it satisfies one of the equivalent conditions of Lemma 1. It is obvious that every subgroup of $G=G_{1} G_{2} \ldots G_{k}$ which contains all factors but one is factorized.

The following lemma studies the behaviour of factorized (prefactorized) subgroups in pairwise mutually permutable products. It is an extension of some known properties of these type of subgroups in the two factors case (see [1, Lemma 1.1.2]).

Lemma 2. Let the group $G=G_{1} G_{2} \ldots G_{k}$ be the pairwise mutually permutable product of the subgroups $G_{1}, G_{2}, \ldots, G_{k}$. Then:

(i) If $S$ is prefactorized in $G$, and $N$ is a normal subgroup of $G$, then $S N / N$ is a prefactorized subgroup of $G / N=\left(G_{1} N / N\right)\left(G_{2} N / N\right) \ldots\left(G_{k} N / N\right)$.

(ii) If $N$ is a prefactorized normal subgroup of $G=G_{1} G_{2} \ldots G_{k}$ and $N$ is contained in $S$, then $S / N$ is a prefactorized subgroup of $G / N=$ $\left(G_{1} N / N\right)\left(G_{2} N / N\right) \ldots\left(G_{k} N / N\right)$ if and only if $S$ is a prefactorized subgroup of $G=G_{1} G_{2} \ldots G_{k}$. 
(iii) If $N$ is a normal subgroup of $G$, a subgroup $S / N$ of the factorized quotient group $G / N=\left(G_{1} N / N\right)\left(G_{2} N / N\right) \ldots\left(G_{k} N / N\right)$ is factorized if and only if $S$ is a factorized subgroup of $G=G_{1} G_{2} \ldots G_{k}$.

(iv) If $U$ is a factorized (prefactorized) subgroup of $G$ and $V$ is a factorized (prefactorized) subgroup of $U$, then $V$ is a factorized (prefactorized) subgroup of $G$.

(v) If $U$ and $V$ are factorized (prefactorized) subgroups of $G$, then also $\langle U, V\rangle$ is factorized (prefactorized).

(vi) If $U$ and $V$ are factorized subgroups of $G$, then $U \cap V$ is a factorized subgroup of $G$.

\section{Proof}

(i) Clearly, $S N / N=\left(S \cap G_{1}\right) N / N \cdot\left(S \cap G_{2}\right) N / N \ldots \cdot\left(S \cap G_{k}\right) N / N \leq$ $\left(S N / N \cap G_{1} N / N\right)\left(S N / N \cap G_{2} N / N\right) \ldots\left(S N / N \cap G_{k} N / N\right)$ which is contained in $S N / N$. This shows that $S N / N$ is prefactorized.

(ii) If $S$ is prefactorized, it is clear by the preceding statement that $S / N$ is prefactorized. Conversely, suppose that $S / N$ is prefactorized. Then using the fact that $N$ is prefactorized and $N \leq S$ we have $S=(S \cap$ $\left.G_{1} N\right)\left(S \cap G_{2} N\right) \ldots\left(S \cap G_{k} N\right)=\left(S \cap G_{1}\right)\left(S \cap G_{2}\right) \ldots\left(S \cap G_{k}\right) N=$ $\left(S \cap G_{1}\right)\left(S \cap G_{2}\right) \ldots\left(S \cap G_{k}\right)\left(N \cap G_{1}\right)\left(N \cap G_{2}\right) \ldots\left(N \cap G_{k}\right)=(S \cap$ $\left.G_{1}\right)\left(S \cap G_{2}\right) \ldots\left(S \cap G_{k}\right) \leq S$, which shows that $S$ is prefactorized.

(iii) Let $S$ be a factorized subgroup of $G$ containing $N$. If $x N=a_{i_{1}} a_{i_{2}} \ldots a_{i_{k}} N$ is an element of $S / N$, with $x \in S$ and $a_{i_{j}} \in G_{i_{j}}$, then $x=a_{i_{1}} a_{i_{2}} \ldots a_{i_{k}} y$, where $y$ belongs to $N \leq S$. Hence $a_{i_{1}} a_{i_{2}} \ldots a_{i_{k}}=x y^{-1}$ belongs to $S$, and so $a_{i_{j}}$ belongs to $S$. Therefore $S / N$ is a factorized subgroup of $G / N$.

Conversely, suppose that the subgroup $S / N$ is factorized in $G / N$. Let $x=a_{i_{1}} a_{i_{2}} \ldots a_{i_{k}}$ be an element of $S$, with $a_{i_{j}} \in G_{i_{j}}$. Since $x N=$ $a_{i_{1}} a_{i_{2}} \ldots a_{i_{k}} N$, it follows that $a_{i_{j}} N$ belongs to $S / N$. Hence $a_{i_{j}}$ belongs to $S$, and so $S$ is factorized.

(iv) Suppose that $U=\left(U \cap G_{1}\right)\left(U \cap G_{2}\right) \ldots\left(U \cap G_{k}\right)$ and $V$ is a prefactorized subgroup of $U$. Then $V=\left(V \cap\left(U \cap G_{1}\right)\right)\left(V \cap\left(U \cap G_{2}\right)\right) \ldots(V \cap(U \cap$ $\left.\left.G_{k}\right)\right)=\left(V \cap G_{1}\right)\left(V \cap G_{2}\right) \ldots\left(V \cap G_{k}\right)$, that is, $V$ is a prefactorized subgroup of $G$. Assume now that $U$ is factorized in $G$ and $V$ is factorized 
in $U$. Then $V$ is prefactorized in $G$. Moreover $G_{i} \cap \prod_{j \neq i} G_{j} \leq U$ and $\left(U \cap G_{i}\right) \cap \prod_{j \neq i}\left(U \cap G_{j}\right) \leq V$. Note that the fact that $U$ is factorized in $G$ yields $\left(U \cap \prod_{j \neq i} G_{j}\right) \leq \prod_{j \neq i}\left(U \cap G_{j}\right)$. Consequently $G_{i} \cap \prod_{j \neq i} G_{j} \leq V$ and $V$ is a factorized subgroup of $G$.

(v) If $U=\left(U \cap G_{1}\right)\left(U \cap G_{2}\right) \ldots\left(U \cap G_{k}\right)$ and $V=\left(V \cap G_{1}\right)\left(V \cap G_{2}\right) \ldots(V \cap$ $\left.G_{k}\right)$, then $\langle U, V\rangle=\left\langle\left(U \cap G_{1}\right)\left(U \cap G_{2}\right) \ldots\left(U \cap G_{k}\right),\left(V \cap G_{1}\right)(V \cap\right.$ $\left.\left.G_{2}\right) \ldots\left(V \cap G_{k}\right)\right\rangle \leq\left\langle\left(\langle U, V\rangle \cap G_{1}\right),\left(\langle U, V\rangle \cap G_{2}\right), \ldots,\left(\langle U, V\rangle \cap G_{k}\right)\right\rangle=$ $\left(\langle U, V\rangle \cap G_{1}\right) \cdot\left(\langle U, V\rangle \cap G_{2}\right) \cdot \ldots \cdot\left(\langle U, V\rangle \cap G_{k}\right) \leq\langle U, V\rangle$. Thus $\langle U, V\rangle$ is a prefactorized subgroup of $G$. Moreover in the case that $U$ and $V$ are factorized subgroups of $G$, we have that $G_{i} \cap \prod_{j \neq i} G_{j} \leq U$ and $G_{i} \cap \prod_{j \neq i} G_{j} \leq V$ for all $i=1,2, \ldots, k$. Consequently $G_{i} \cap \prod_{j \neq i} G_{j} \leq$ $\langle U, V\rangle$ for all $i=1,2, \ldots, k$ and $\langle U, V\rangle$ is a factorized subgroup of $G$.

(vi) If $a_{i_{1}} a_{i_{2}} \ldots a_{i_{k}} \in U \cap V$, with $a_{i_{j}} \in G_{i_{j}}$, where $\left\{i_{1}, i_{2}, \ldots, i_{k}\right\}=\{1,2, \ldots, k\}$, then using the fact that $U$ and $V$ are factorized, we have that $a_{i_{j}}$ belongs to $U \cap V$ for all $i_{j} \in\{1,2, \ldots, k\}$. Therefore applying Lemma $1(\mathrm{i}), U \cap V$ is a factorized subgroup of $G$.

\section{Normal prefactorized subgroups}

We begin with some prominent conjugacy classes of subgroups for a positive statement.

Proposition 1. (i) Let the group $G=G_{1} G_{2} \ldots G_{k}$ be a product of pairwise mutually permutable subgroups. For each prime $p$ dividing $|G|$ there exists a Sylow p-subgroup of $G, P$ say, such that $P$ is prefactorized, that is, $P=\left(P \cap G_{1}\right)\left(P \cap G_{2}\right) \ldots\left(P \cap G_{k}\right)$. Moreover $P \cap G_{i}$ is a Sylow p-subgroup of $G_{i}$ for all $i=1,2, \ldots, k$.

(ii) Let the group $G=G_{1} G_{2} \ldots G_{k}$ be a product of pairwise mutually permutable soluble subgroups. For each set of primes $\pi$ dividing the order of $G$, there exists a Hall $\pi$-subgroup of $G, H$ say, such that $H$ is prefactorized, that is, $H=\left(H \cap G_{1}\right)\left(H \cap G_{2}\right) \ldots\left(H \cap G_{k}\right)$. Moreover $H \cap G_{i}$ is a Hall $\pi$-subgroup of $G_{i}$ for all $i=1,2, \ldots, k$.

Proof We prove (ii). Since all $G_{i}$ are soluble, applying [5, Theorem 1] $G$ is soluble. We proceed by induction on $k$. The result is clear if $k=1$. Suppose that $k>1$ and the result is true for all groups which are pairwise 
mutually permutable products of less than $k$ factors. Consider the product $G_{1} G_{2} \ldots G_{k-1}$. Then there exists a Hall $\pi$-subgroup $T$ of $G_{1} G_{2} \ldots G_{k-1}$ such that $T=\left(T \cap G_{1}\right)\left(T \cap G_{2}\right) \ldots\left(T \cap G_{k-1}\right)$. Moreover $T \cap G_{i}$ is a Hall $\pi$ subgroup of $G_{i}$ for all $i=1,2, \ldots, k-1$. Since the product is pairwise mutually permutable, it follows that $T G_{k}$ is a subgroup of $G$. Let $H$ be a Hall $\pi$-subgroup of $T G_{k}$ containing $T$. Then $H=H \cap T G_{k}=T\left(H \cap G_{k}\right)$. Also $T \cap G_{i} \leq H \cap G_{i}$ for all $i=1,2, \ldots k-1$. But $T \cap G_{i}$ is a Hall $\pi$ subgroup of $G_{i}$ for all $i=1,2, \ldots k-1$. Therefore $T \cap G_{i}=H \cap G_{i}$ for all $i=1,2, \ldots k-1$ and $H=\left(H \cap G_{1}\right)\left(H \cap G_{2}\right) \ldots\left(H \cap G_{k}\right)$. Note that $\left|T G_{k}: H\right|=\left|G_{k}: H \cap G_{k}\right|$ is a $\pi^{\prime}$-number. Consequently $\left(H \cap G_{k}\right)$ is a Hall $\pi$-subgroup of $G_{k}$. Now an argument on the orders shows that $H$ is a Hall $\pi$-subgroup of $G$.

Using the same arguments as above we obtain (i) for non-soluble groups.

The following example shows that in a mutually permutable product (even if it is a totally permutable one) there is not necessarily a factorized Sylow subgroup for each prime $p$ dividing the order of $G$.

Example 2 Let $X=\left\langle x, y \mid x^{3}=y^{2}=1, x^{y}=x^{-1}\right\rangle$ be a group isomorphic to the symmetric group of degree 3 and $Y=\left\langle a, b \mid a^{5}=b^{2}=[a, b]=1\right\rangle \simeq$ $C_{5} \times C_{2}$. Consider $G=X \times Y, A=\langle y\rangle \times\langle a\rangle \times\langle b\rangle$ and $B=\langle x\rangle \times\langle b\rangle$. Then $G=A B$ is the totally permutable product of $A$ and $B$. Moreover $A \cap B=\langle b\rangle$ and $\langle x\rangle$ is Sylow 3 subgroup of $G$ which is clearly prefactorized but not factorized.

Remark Assume that a certain normal subgroup $N$ of a pairwise mutually permutable product $G=G_{1} G_{2} \ldots G_{k}$ satisfies that $N / M$ is prefactorized in $G / M=\left(G_{1} M / M\right)\left(G_{2} M / M\right) \ldots\left(G_{k} M / M\right)$ for each minimal normal subgroup $M$ of $G$ contained in $N$. Then either $N$ is prefactorized in $G=G_{1} G_{2} \ldots G_{k}$ or $N$ is a minimal normal subgroup of $G$.

Assume that $N$ is not prefactorized in $G$. Then $N \neq 1$. If $N$ is not a minimal normal subgroup of $G$, there exists a minimal normal subgroup $M$ of $G$ with $M \leq N$. By hypothesis, $N / M$ is prefactorized in $G / M$, that is, $N / M=\left(\left(G_{1} M / M\right) \cap(N / M)\right)\left(\left(G_{2} M / M\right) \cap(N / M)\right) \ldots\left(\left(G_{k} M / M\right) \cap(N / M)\right)$ and from here $N=\left(N \cap G_{1}\right)\left(N \cap G_{2}\right) \ldots\left(N \cap G_{k}\right) M$. On the other hand, by [5, Lemma 1(ii)], $\left(N \cap G_{1}\right)\left(N \cap G_{2}\right) \ldots\left(N \cap G_{k}\right)$ is a normal subgroup of $G$. If $\left(N \cap G_{1}\right)\left(N \cap G_{2}\right) \ldots\left(N \cap G_{k}\right) \neq 1$, then it contains a minimal normal 
subgroup of $G, R$ say, and this implies $N=\left(N \cap G_{1}\right)\left(N \cap G_{2}\right) \ldots\left(N \cap G_{k}\right)$, that is, $N$ is prefactorized, contrary to assumption. Therefore we may assume that $\left(N \cap G_{1}\right)\left(N \cap G_{2}\right) \ldots\left(N \cap G_{k}\right)=1$. Thus $N=M$ and $N$ is a minimal normal subgroup of $G$.

We present now an example of a $p$-group in which the derived subgroup is not prefactorized.

Example 3 Consider the group $M=(\langle a\rangle \times\langle b\rangle \times\langle x, y\rangle) / N$ with $N=$ $\left\langle a^{2} b^{2} x^{2}\right\rangle, a$ and $b$ elements of order 4 and $\langle x, y\rangle \simeq Q_{8}$, the quaternion group of order 8 . Denote by $u^{*}$ the class $u N$ of $M$. We show that $M=$ $\left\langle a^{*}\right\rangle\left\langle a^{*} x^{*}\right\rangle\left\langle b^{*}\right\rangle\left\langle b^{*} y^{*}\right\rangle$ is a pairwise mutually permutable product: Since two of the factors are normal subgroups, we have to check only one case, and we obtain $\left(a^{*} x^{*}\right)\left(b^{*} y^{*}\right)=a^{*} b^{*} x^{*} y^{*}=a^{*} b^{*} y^{*} x^{*}\left(a^{*} b^{*}\right)^{2}=b^{*} y^{*} a^{*} x^{*}\left(b^{*} y^{*}\right)^{2}\left(a^{*} x^{*}\right)^{2}=$ $\left(b^{*} y^{*}\right)^{-1}\left(a^{*} x^{*}\right)^{-1}$ since $\left(a^{*} x^{*}\right)^{2}=\left(b^{*}\right)^{2}$ and $\left(b^{*} y^{*}\right)^{2}=\left(a^{*}\right)^{2}$. Note that all squares belong to the center of $M$, so given relation leads to all the other further relations to be checked, so if $\{e, f\} \subseteq\{1,-1\}$ we find $\left(a^{*} x^{*}\right)^{e}\left(b^{*} y^{*}\right)^{f}=$ $\left(b^{*} y^{*}\right)^{-f}\left(a^{*} x^{*}\right)^{-e}$. Note the subgroup $M^{\prime}=M^{\prime} M^{4}$ is not prefactorized.

We will now exhibit some positive results.

Theorem 1. Let the group $G=G_{1} G_{2} \ldots G_{k}$ be the product of the pairwise mutually permutable subgroups $G_{1}, G_{2}, \ldots, G_{k}$. Then

(i) The subgroup of $G$ which is generated by all $\Pi$-elements of $G$ (where $\Pi$ denotes a set of primes dividing the order of $G$ ) is prefactorized in $G$.

(ii) $G^{p}=\left\langle x^{p} \mid x \in G\right\rangle$ is prefactorized in $G$ for each prime $p$.

(iii) If $M$ and $N$ are prefactorized normal subgroups of $G$ and $N$ has exponent $p, p$ a prime, then also $[M, N]$ is prefactorized in $G$.

\section{Proof}

(i) Denote by $N$ the subgroup generated by all $\Pi$-elements of $G$, $\Pi$ a set of primes dividing the order of $G$. Assume that $N$ is not prefactorized and choose for $G$ a couterexample of minimal order. Then $N / M$ is prefactorized in $G / M=\left(G_{1} M / M\right)\left(G_{2} M / M\right) \ldots\left(G_{k} M / M\right)$ for each minimal normal subgroup $M$ of $G$ contained in $N$. By the above Remark, $N$ is a minimal normal subgroup of $G$ and so $G_{i} \cap N=1$ for 
all $i=1,2, \ldots, k$. Since $G / N$ is a $\Pi^{\prime}$-group, it follows that $G_{i} N / N$ is also a $\Pi^{\prime}$-group for all $i$. Therefore $G$ is a $\Pi^{\prime}$-group. This inplies that $N=1$, contrary to assumption. Hence $N$ is prefactorized.

(ii) Let $N=G^{p}$. Assume that $N \neq\left(N \cap G_{1}\right)\left(N \cap G_{2}\right) \ldots\left(N \cap G_{k}\right)$ and let $G$ be of minimal order. Arguing as in (i), $N$ is a minimal normal subgroup of $G$ and so $N \cap G_{i}=1$ for all $i=1,2, \ldots, k$. In particular, $G_{i}$ have exponent $p$ for all $i$. Hence $G$ is a $p$-group and $|N|=p$. Choose $x \in G_{j}$. Then $\langle x\rangle G_{i}=G_{i}\langle x\rangle$ and $G_{i}$ is a subgroup of index at most $p$ in the product $G_{i}\langle x\rangle$. Thus $G_{i}$ is normalized by all elements $x$ of $G_{j}$ and so by $G$.

Let $t$ be an element of $G$. Then $t=u_{1} u_{2} \ldots u_{k}$ with $u_{i} \in G_{i}, i=$ $1,2, \ldots, k$. Suppose that $t^{p} \neq 1$ and choose $t$ with least number of nontrivial factors. Let $u_{w}$ be the first nontrivial factor. Then $t=u_{w} s$ and $s^{p}=1$ by our choice of $t$. Since $G_{w}$ is normal in $G$, it follows that every conjugate of $u_{w}$ belong to $G_{w}$. We have $t^{p}=\left(u_{w} s\right)^{p} s^{-p}=$ $u_{w}\left(s u_{w} s^{-1}\right)\left(s^{2} u_{w} s^{-2}\right) \ldots\left(s^{p-1} u_{w} s^{1-p}\right) \in G_{w}$. This means that $t^{p} \in$ $N \cap G_{w}=1$, against the choice of $t$. Therefore $G$ has exponent $p$ and $N=G^{p}=1$, the final contradiction.

(iii) Assume the result is not true and let $G$ be a minimal counterexample. By Lemma 2(v), $M N$ is a prefactorized subgroup of $G$. If we suppose that $M N$ is a proper subgroup of $G$, then it is clear that $M N$ satisfies the same hypotheses as $G$. By the choice of $G,[M, N]$ is prefactorized in $M N$. Lemma 2(iv) yields $[M, N]$ is prefactorized in $G$. Therefore we may assume that $G=M N$. Then $[M, N]$ is a normal subgroup of $G$ and by the remark we may assume that $[M, N]$ is a minimal normal subgroup of $G$ such that $[M, N] \cap G_{i}=1$ for every $i=1,2, \ldots, k$. Let $1 \neq x \in N \cap G_{j}$. Then $x$ has order $p$ since $N^{p}=1$. In particular, it normalizes $G_{i}$ since $\left|G_{i}\langle x\rangle: G_{i}\right| \in\{1, p\}$. This implies that $N \cap$ $G_{j}$ normalizes $M \cap G_{i}$ for all $i=1,2, \ldots, k$. On the other hand, by hypotesis we have $M=\left(M \cap G_{1}\right)\left(M \cap G_{2}\right) \ldots\left(M \cap G_{k}\right)$ and $N=(N \cap$ $\left.G_{1}\right)\left(N \cap G_{2}\right) \ldots\left(N \cap G_{k}\right)$. Hence $[M, N]=\left[\left(N \cap G_{1}\right)\left(N \cap G_{2}\right) \ldots(N \cap\right.$ $\left.\left.G_{k}\right),\left(M \cap G_{1}\right)\left(M \cap G_{2}\right) \ldots\left(M \cap G_{k}\right)\right]$. Applying [11, A; 7.4(f)], $[M, N]=$ $\left[N \cap G_{1}, M\right]\left[N \cap G_{2}, M\right] \ldots\left[N \cap G_{k}, M\right]$. Let $z \in\left[N \cap G_{j}, M\right]$. By [11, A; 7.2], $z=x_{1} x_{2} \ldots x_{k}$ with $x_{i} \in\left[N \cap G_{j}, M \cap G_{i}\right]^{g}, g \in G$. Therefore $x_{i} \in G_{i}^{g} \cap[N, M]$. Now $x_{i}^{g^{-1}} \in G_{i} \cap[N, M]=1$ and $x_{i}=1$. Consequently $z=1$ and from here $[M, N]=1$. This final contradiction proves the 
claim.

Corollary 1. (i) If $G=G_{1} G_{2} \ldots G_{k}$ is a pairwise mutually permutable product of exponent $p$, then every term of the descending central series and every term of the derived series of $G$ is prefactorized in $G$. Furthermore for every prefactorized normal subgroup $M$ of $G$, we have that $[M, G]$ and $M^{\prime}$ are prefactorized subgroups of $G$.

(ii) If $G=G_{1} G_{2} \ldots G_{k}$ is a pairwise mutually permutable product, then $G^{p} G^{\prime}$ is prefactorized in $G$.

(iii) If $G=G_{1} G_{2} \ldots G_{k}$ is a pairwise mutually permutable product and $m$ is a squarefree integer, then $G^{m} G^{\prime}$ is prefactorized in $G$.

Proof (i) is a direct consequence of part (iii) of Theorem 1.

To prove (ii), notice that applying (i), $\left(G / G^{p}\right)^{\prime}=G^{p} G^{\prime} / G^{p}$ is prefactorized in $G / G^{p}$ and $G^{p}$ is prefactorized in $G$ by Theorem 1(ii). The result now follows from Lemma 2(ii).

We proceed by induction on the number $k$ of prime factors of $m$ for statement (iii). The result is true if $m$ is a prime by (ii). Assume the result is true for all squarefree integers which are products of $k-1$ primes and choose a prime $p$ dividing $m$. Then $m=p n$ and $H_{n}=G^{n} G^{\prime}$ and $H_{p}=G^{p} G^{\prime}$ are prefactorized by the induction hypothesis. Hence $\left(H_{n}\right)^{p}\left(H_{n}\right)^{\prime}$ and $\left(H_{p}\right)^{n}\left(H_{p}\right)^{\prime}$ are prefactorized in $G$. Now we have the following inclusions:

$$
\begin{aligned}
& G^{m}\left(G^{\prime}\right)^{p} \subseteq\left(H_{n}\right)^{p}\left(H_{n}\right)^{\prime} \subseteq G^{m} G^{\prime} \\
& G^{m}\left(G^{\prime}\right)^{n} \subseteq\left(H_{p}\right)^{n}\left(H_{p}\right)^{\prime} \subseteq G^{m} G^{\prime}
\end{aligned}
$$

and therefore $G^{m} G^{\prime}=\left(\left(H_{n}\right)^{p}\left(H_{n}\right)^{\prime}\left(H_{p}\right)^{n}\left(H_{p}\right)^{\prime}\right)$ is prefactorized in $G$ by Lemma $2(\mathrm{v})$.

Note that the hypothesis about $m$ in the above corollary is essential (see Example 3).

The following statement gives an indication that there is quite a range of prefactorized normal subgroups.

Corollary 2. Let the soluble group $G=G_{1} G_{2} \ldots G_{k}$ be a pairwise mutually permutable product of the subgroups $G_{1}, G_{2}, \ldots, G_{k}$. Then there is a descending sequence of characteristic prefactorized subgroups $G=A_{0} \supset A_{1} \supset A_{2} \supset$ $\ldots \supset A_{m}=1$ such that every quotient $A_{s} / A_{s+1}$ is an elementary abelian p-group. 
Proof Since $G \neq 1$ is soluble, we have $G^{\prime} \neq G$. Choose a prime dividing $\left|G / G^{\prime}\right|$. Then also $G^{p} G^{\prime} \neq G$ and we may take $A_{1}=G^{p} G^{\prime}$ by the statement (ii) of the above result. The corollary now follows by induction on $|G|$.

The following result shows that $\mathcal{F}$-residuals, for $\mathcal{F}$ a saturated formation of soluble groups, have a good behaviour concerning pairwise mutually permutable products and prefactorizations.

Theorem 2. Let the group $G=G_{1} G_{2} \ldots G_{k}$ be the product of the pairwise mutually permutable subgroups $G_{1}, G_{2}, \ldots, G_{k}$. If $\mathcal{F}$ is a saturated formation of soluble groups, then $G^{\mathcal{F}}$, the $\mathcal{F}$-residual of $G$, is prefactorized.

Proof Denote by $N=G^{\mathcal{F}}$ the $\mathcal{F}$-residual of $G$. Assume the result is not true and let $G$ be a counterexample of minimal order. By the remark we may assume that $N$ is a minimal normal subgroup of $G$ and $N \cap G_{i}=1$ for all $i=1,2, \ldots, k$. Since $G / N$ is soluble, we that $G_{i} \simeq G_{i} N / N$ are soluble and so $G$ is soluble by [5, Theorem 1]. Then $N$ is an elementary abelian $p$-group for some prime $p$. Let $F$ denote the canonical local definition of $\mathcal{F}$, that is, the uniquely determined function defining $\mathcal{F}$ which is integrated and full, that is, $F(q) \subseteq \mathcal{F}$ and $F(q)=\mathcal{S}_{q} F(q)$ for all primes $q$ (see [11, IV; 3.9]). Clearly $G^{\mathcal{F}}$ is contained in $L=G^{F(p)}$, the $F(p)$-residual of $G$ and $L / N$ is $p$-nilpotent by $[11, \mathrm{IV} ; 3.2(\mathrm{~b})]$. Denote by $M / N$ the normal Hall $p^{\prime}$-subgroup of $L / N$. By Proposition 1 (ii), there exist a prefactorized Hall $p^{\prime}$-subgroup $H$ of $G$. Then $H=\left(H \cap G_{1}\right)\left(H \cap G_{2}\right) \ldots\left(H \cap G_{k}\right)$ is a product of pairwise mutually permutable subgroups, $G_{i} H$ is a subgroup of $G$ and the Sylow $p$-subgroups of $G_{i}$ are Sylow $p$-subgroups of $G_{i} H$. Now $H G_{i} \cap N$ is a normal p-subgroup of $H G_{i}$ and so it is contained in every Sylow $p$-subgroup of $H G_{i}$. In particular it is contained in every Sylow $p$-subgroup of $G_{i}$. Consequently $H G_{i} \cap N=1$. Now $M \cap H$ is a Hall $p^{\prime}$-subgroup of $M$. Since $M / N$ is a $p^{\prime}$-group, it follows that $M=(M \cap H) N$. Then $M \cap H G_{i}=(M \cap H) N \cap H G_{i}=(M \cap H)\left(N \cap H G_{i}\right)=M \cap H$. This implies that $M \cap H$ is normalized by all $G_{i}$ and therefore also by $G$. Therefore $M=(M \cap H) \times N$ and $M \leq C_{G}(N)$. Since $N$ is a minimal normal $p$-subgroup of $G$, we have that $G / C_{G}(N)$ does not possess nontrivial normal $p$-subgroups. Consequently $L C_{G}(N) / C_{G}(N)=1$, that is, $L \leq C_{G}(N)$. We have that $G / N$ is an $\mathcal{F}$-group and $G / C_{G}(N) \in F(p)$. Therefore applying [11, IV; 3.2], $G \in \mathcal{F}$ and $N=1$. This is a contradiction. 
Unfortunately $\mathcal{F}$-residuals, even for subgroup-closed saturated formations, are not necessarily factorized subgroups in the group as the following example shows:

Example 4 Consider the group $G$ as in Example 2. Then it is clear that the nilpotent residual of $G, G^{\mathcal{N}}$, is equal to $\langle x\rangle$ which is clearly prefactorized in $G$. However $A \cap B=\langle b\rangle$ is not contained in $G^{\mathcal{N}}$.

\section{Prefactorized projectors and normalizers}

¿From now on all groups considered will be finite and soluble.

In this section we analyze the behaviour of projectors and normalizers associated to saturated formations in pairwise totally and mutually permutable products.

Lemma 3. Let the group $G=G_{1} G_{2} \ldots G_{k}$ be the pairwise totally permutable product of the subgroups $G_{1}, G_{2}, \ldots, G_{k}$. If $G$ is a primitive group, then either $G$ is supersoluble or $\prod_{i \neq j} G_{j}=1$ for some $i \in\{1,2, \ldots, k\}$.

Proof Let $N$ be the unique minimal normal subgroup of $G$. We know that $N$ is abelian and $C_{G}(N)=N$. Applying [4, Lemma 1], the supersoluble residual $\left(G_{i}\right)^{\mathcal{U}}$ of $G_{i}$ is a normal subgroup of $G$ for all $i=1,2, \ldots, k$. If $\left(G_{i}\right)^{\mathcal{U}}=1$ for all $i \in\{1,2, \ldots, k\}$, then $G$ is supersoluble by [4, Theorem 1]. Hence we may assume without loss of generality that $\left(G_{1}\right)^{\mathcal{U}} \neq 1$. Then $N$ is contained in $\left(G_{1}\right)^{\mathcal{U}}$. Applying [3, Corollary], $G_{2} \ldots G_{k}$ centralizes $\left(G_{1}\right)^{\mathcal{U}}$. Therefore $G_{2} \ldots G_{k} \leq C_{G}(N)=N \leq G_{1}$. Consequently $G=G_{1}$. This implies that, if $i \neq 1$, all subgroups of $G_{i}$ are permutable in $G$. Assume that $G_{i} \neq 1$ for some $i \in\{2,3, \ldots, k\}$ and consider a cyclic subgroup $W$ of prime order of $G_{i}$. Then, if $K$ is a complement of $N$ in $G$, we have that $W K$ is a subgroup of $G$ and $W=N \cap W K$ is a normal subgroup of $G$. The minimality of $N$ implies that $N=W$ and $G$ is supersoluble, contrary to assumption. Consequently $G_{i}=1$ for all $i \in\{2,3, \ldots, k\}$.

Lemma 4. Let the group $G=G_{1} G_{2} \ldots G_{k}$ be the pairwise totally permutable product of the subgroups $G_{1}, G_{2}, \ldots, G_{k}$. If $G$ is supersoluble and it is a primitive group with unique minimal normal subgroup $N$, then one of the following cases holds: 
(i) If $N \leq G_{i}$ for all $i \in\{1,2, \ldots, k\}$ then there exists $r \in\{1,2, \ldots, k\}$ such that $G=G_{r} \neq N$ and either $G_{i}=N$ for all $i \neq r$ or $G=G_{1}=$ $G_{2}=\ldots=G_{k}=N$.

(ii) If $N \leq G_{i}$ for $i=1,2, \ldots, r, r<k$, and $G_{j} \neq 1$, for some $j>r$, then $G_{1}=G_{2}=\cdots=G_{r}=N$ and $G_{r+1} G_{r+2} \ldots G_{k} \neq 1$ is a complement of $N$ in $G$.

(iii) If $N \leq G_{i}$ for $i=1,2, \ldots, r, k>r \geq 2$ and $G_{j}=1$ for all $j>r$, then there exists $1 \leq s \leq r$ such that $G=G_{s} \neq N$ and either $G_{i}=N$ for all $s \neq i \leq r$, or $G=G_{1}=G_{2}=\ldots=G_{r}=N$.

(iv) There exists $i \in\{1,2, \ldots, k\}$ such that $G=G_{i}$ and $G_{j}=1$ for all $j \neq i$.

Proof Let $p$ be the prime dividing $|N|$. Since $G$ is supersoluble, we have that $N$ is of order $p$ and $C_{G}(N)=N=\langle x\rangle$ is the Sylow $p$-subgroup of $G$. Let $M$ denote a core-free complement of $N$ in $G$. Then $M=\langle y\rangle$ is an abelian maximal subgroup of $G$ with exponent dividing $p-1$. In particular, $M$ is a Hall $p^{\prime}$-subgroup of $G$. Therefore $N$ has to be contained in at least one of the factors $G_{i}, i=1,2, \ldots, k$.

(i) Assume first that $N \leq G_{i}$ for all $i \in\{1,2, \ldots, k\}$. Consider one of the factors $G_{i}$ and take $G_{j}$ with $G_{j} \neq G_{i}$. We see that $G_{i}=N$ or $G_{j}=N$. It is clear that $G_{i}=\langle x\rangle\left\langle y^{\alpha_{i}}\right\rangle$ and in the same way $G_{j}=\langle x\rangle\left\langle y^{\alpha j}\right\rangle$ with $\left\langle y^{\alpha_{i}}\right\rangle \neq\left\langle y^{\alpha j}\right\rangle$. Suppose that $y^{\alpha_{i}} \neq 1 \neq y^{\alpha_{j}}$. Write $H=\left\langle x y^{\alpha_{i}}\right\rangle$. If $p$ divides the order of $H$, then $N \leq H$. Since $H$ is abelian, $H=N$ and so $y^{\alpha_{i}} \in N$ and $G_{i}=N$. This contradiction implies that $H=\left\langle x y^{\alpha_{i}}\right\rangle$ is a $p^{\prime}$-group. Now the fact that $G_{i}$ and $G_{j}$ are totally permutable yields that $\left\langle x y^{\alpha_{i}}, y^{\alpha_{j}}\right\rangle=\left\langle x y^{\alpha_{i}}\right\rangle\left\langle y^{\alpha_{j}}\right\rangle$ is a $p^{\prime}$-group and therefore it is abelian. Consequently $\left[x y^{\alpha_{i}}, y^{\alpha_{j}}\right]=1$. Moreover $\left[y^{\alpha_{i}}, y^{\alpha_{j}}\right]=1$. Let $s=y^{\alpha_{i}}$. By $[11, \mathrm{~A} ; 7.2(\mathrm{c})], 1=\left[x y^{\alpha_{i}}, y^{\alpha_{j}}\right]=\left[x, y^{\alpha_{j}}\right]^{s}\left[y^{\alpha_{i}}, y^{\alpha_{j}}\right]=\left[x, y^{\alpha_{j}}\right]^{s}$. Hence $y^{\alpha_{j}}=1$, which contradicts our assumption. Therefore if $G_{i} \neq G_{j}$ we have either $G_{i}=N$ or $G_{j}=N$. Assume that $M \neq 1$. Then at least one of the factors has a non-trivial Hall $p^{\prime}$-subgroup. Without loss of generality, we may suppose that $M \cap G_{1} \neq 1$. The above argument implies that either $G_{i}=G_{1}$ or $G_{i}=N$ for every $i \neq 1$. Assume that $G_{j}=G_{1}$ for some $j \neq 1$. Applying the above argument, $1 \neq y^{\alpha_{j}}$ centralizes $x$. This contradiction shows that $G_{2}=\ldots=G_{k}=N$ and then $G=G_{1}$. 
(ii) Suppose that $N \leq G_{i}$ for $i=1,2, \ldots, r, r<k$. Then $N \cap G_{j}=1$ for all $j>r$. Assume that $G_{j} \neq 1$, for some $j>r$. Then $G_{j}=\langle z\rangle$ is a nontrivial $p^{\prime}$-subgroup of $G$. Suppose there exists $i \leq r$ such that $G_{i} \neq N$. Then $G_{i}=N\left\langle y^{\alpha_{i}}\right\rangle$ and $y^{\alpha_{i}} \neq 1$. Consider $H=\left\langle x y^{\alpha_{i}}\right\rangle$. Arguing as in (i), $H$ is a $p^{\prime}$-group. Moreover as $G_{i}$ and $G_{j}$ are totally permutable, $H$ permutes with $G_{j}$ and $H G_{j}$ is a $p^{\prime}$-subgroup of $G$ and hence $H G_{j}$ is abelian. Therefore $\left[x y^{\alpha_{i}}, z\right]=1$. It is also clear that $\left\langle y^{\alpha_{i}}\right\rangle$ permutes with $\langle z\rangle$. Therefore $\left\langle y^{\alpha_{i}}\right\rangle\langle z\rangle$ is an abelian $p^{\prime}$-group. Hence $\left[y^{\alpha_{i}}, z\right]=1$. Therefore $z$ centralizes $x$. This contradiction yields $G_{i}=N$ for all $i=1,2, \ldots, r$. Therefore $G_{r+1} G_{r+2} \ldots G_{k}$ is a complement of $N$ in $G$.

(iii) If $N \leq G_{i}$ for $i=1,2, \ldots, r, k>r \geq 2$, and $G_{j}=1$ for all $j>r$, then we are in case (i). Hence there exists $1 \leq s \leq r$ such that $G=G_{s} \neq N$ and $G_{i}=N$ for all $s \neq i \leq r$, or $G=G_{1}=G_{2}=\ldots=G_{r}=N$.

The remaining possibility is case (iv).

Definition 1. If $\mathcal{X}$ is a class of groups, a maximal subgroup $M$ of a group $G$ is called $\mathcal{X}$-abnormal in $G$ if $G /$ Core $_{G}(M)$ does not belong to $\mathcal{X}$. A subgroup $S$ is called sub-X-abnormal in $G$ if either $G=S$ or there exists a chain

$$
S=S_{0} \leq S_{1} \leq \ldots \leq S_{n}=G
$$

with $S_{i}$ an $\mathcal{X}$-abnormal maximal subgroup of $S_{i+1}$ for all $i=0,1, \ldots, n-1$.

Theorem 3. Let the group $G=G_{1} G_{2} \ldots G_{k}$ be the pairwise totally permutable product of the subgroups $G_{1}, G_{2}, \ldots, G_{k}$. If $\mathcal{F}$ is a saturated formation containing the class $\mathcal{U}$ of all supersoluble groups, then every sub- $\mathcal{F}$ abnormal subgroup of $G$ is factorized.

Proof. We proceed by induction on the index $|G: S|$ of $S$ in $G$. Suppose that $S$ is a maximal $\mathcal{F}$-abnormal subgroup of $G$. If $\operatorname{Core}_{G}(S)=1$, then, by Lemma $3, G$ is either supersoluble or there exists $i \in\{1,2, \ldots, k\}$ such that $\prod_{i \neq j} G_{j}=1$ and $G=G_{i}$. Since $\mathcal{F}$ contains $\mathcal{U}$ and $S$ is $\mathcal{F}$ abnormal, it follows that $G$ is not supersoluble. Hence $G=G_{i}$ for some $i$. It is clear that in this case $S$ is a factorized subgroup of $G$. Assume that $D=\operatorname{Core}_{G}(S) \neq 1$. As $G / D$ does not belong to $\mathcal{F}$, it cannot be supersoluble. Applying now Lemma 3 to $G / D=\left(G_{1} D / D\right)\left(G_{2} D / D\right) \ldots\left(G_{k} D / D\right)$ we obtain that there exists $i \in\{1,2, \ldots, k\}$ (we can assume without loss of generality that $i=1$ ) such that $G_{2} G_{3} \ldots G_{k}$ is contained in $D$. Then 
$S=G_{2} G_{3} \ldots G_{k}\left(S \cap G_{1}\right)=\left(S \cap G_{2}\right)\left(S \cap G_{3}\right) \ldots\left(S \cap G_{k}\right)\left(S \cap G_{1}\right)$ and $G_{i} \cap \prod_{j \neq i} G_{j} \leq S$ for all $i=1,2, \ldots, k$. Hence $S$ is factorized.

Assume now that $S$ is not a maximal subgroup of $G$ and let $S_{1}$ be an $\mathcal{F}$-abnormal maximal subgroup of $G$ containing $S$ such that $S$ is sub- $\mathcal{F}$ abnormal in $S_{1}$. Then $S_{1}$ is a factorized subgroup of $G$ and $\left|S_{1}: S\right|<|G: S|$. The induction hypothesis implies that $S$ is a factorized subgroup of $S_{1}$. Then $S$ is factorized in $G$ by Lemma 2(iv).

Applying [11, IV; 5.11] and [11, V; 3.10], $\mathcal{F}$-projectors and $\mathcal{F}$-normalizers associated to a saturated formation $\mathcal{F}$ are sub- $\mathcal{F}$-abnormal subgroups. Hence we have:

Corollary 3. Let the group $G=G_{1} G_{2} \ldots G_{k}$ be a pairwise totally permutable product of the subgroups $G_{1}, G_{2}, \ldots, G_{k}$. If $\mathcal{F}$ is a saturated formation containing the class $\mathcal{U}$ of all supersoluble groups, then the $\mathcal{F}$-projectors and $\mathcal{F}$ normalizers of $G$ are factorized subgroups of $G$.

For the saturated formation $\mathcal{N}$ of nilpotent groups we have a much weaker statement.

Theorem 4. Let the group $G=G_{1} G_{2} \ldots G_{k}$ be the pairwise totally permutable product of the subgroups $G_{1}, G_{2}, \ldots, G_{k}$. Then there is a prefactorized Carter subgroup of $G$.

Proof Assume the result is not true and let $G$ be a counterexample with $|G|+\left|G_{1}\right|+\left|G_{2}\right|+\ldots+\left|G_{k}\right|$ minimal. If $G$ is a nilpotent group, then $G$ is its own Carter subgroup and the conclusion follows. Assume $G$ is not nilpotent and let $C$ denote a Carter subgroup of $G$. Then there exists a non-normal maximal subgroup $M$ of $G$ with $C \leq M$. Denote by $K=\operatorname{Core}_{G}(M)$. If $K=1$, then $G$ is a primitive group. By Lemma 3, there exists $i \in\{1,2, \ldots, k\}$ such that $\prod_{j \neq i} G_{j}=1$ and $G=G_{i}$ or $G$ is supersoluble. In the first case, it is clear that $C$ is prefactorized. If $G$ is supersoluble, then $G=N M$ with $N=C_{G}(N)=G^{\mathcal{N}}$ the unique minimal normal subgroup of $G,|N|=p, p$ a prime, and $M$ an abelian complement of $N$ with trivial core. By [11, IV; 5.18], the Carter subgroups of $G$ are the complements of $N$ in $G$. Applying Lemma $4, C$ is prefactorized. This contradiction yields $K \neq 1$. Then $G / K=(N / K)(M / K)$ is a primitive group, $N / K$ is the unique minimal normal subgroup of $G / K$, $C_{G / K}(N / K)=N / K$ and $M / K$ is a maximal subgroup of $G / K$ with trivial 
core. Also $G / K$ is the pairwise totally permutable product of the subgroups $G_{1} K / K, G_{2} K / K, \ldots, G_{k} K / K$. Applying Lemma $3, G / K$ is either supersoluble or there exists $i \in\{1,2, \ldots, k\}$ (we can assume without loss of generality that $i=1$ ), such that $G_{2} G_{3} \ldots G_{k} \leq K \leq M$. Assume that $G / K$ is not supersoluble. Then $M=G_{2} G_{3} \ldots G_{k}\left(M \cap G_{1}\right)$ is a pairwise totally permutable product. By the choice of $G, M$ has a prefactorized Carter subgroup. But $C$ is a Carter subgroup of $M$. Therefore there exists $m \in M$ such that $C^{m}$ is prefactorized in $M$. By Lemma 2(iv), $C^{m}$ is prefactorized in $G$. Consequently we may assume that $G / K$ is supersoluble. In particular, $|N / K|=p$ and $M / K$ is abelian of exponent dividing $p-1$.

On the other hand, applying [5, Lemma 1], $W=\left(K \cap G_{1}\right)\left(K \cap G_{2}\right) \ldots(K \cap$ $\left.G_{k}\right)$ is a normal subgroup of $G$. If $W \neq 1$, then the choice of $G$ implies that $G / W$ has a prefactorized Carter subgroup, $C^{g} W / W$, for some $g \in G$. Since $W$ is prefactorized, it follows that $C^{g} W$ is prefactorized in $G$ by Lemma 2(ii). Now $C^{g} W$ is a proper subgroup of $G$ because it is contained in $M^{g}$. Clearly $C^{g}$ is a Carter subgroup of $C^{g} W$. By the minimal choice of $G$, there exists $t \in C^{g} W$ such that $C^{g t}$ is prefactorized in $C^{g} W$. Lemma 2(iv) implies that $C^{g t}$ is prefactorized in $G$. Consequently we may assume that $W=1$, that is, $K \cap G_{i}=1$ for all $i=1,2, \ldots, k$. It now follows that $G_{i}$ is supersoluble so that $G$ is also supersoluble by Theorem 3 of [4].

It is clear that $p$ is the largest prime dividing the order of $G / K$. Assume $p$ is not the largest prime dividing the order of $G$ and let $Q \neq 1$ denote a Sylow $q$-subgroup of $G$ with $q$ the largest prime divisor of the order of $G, q \neq p$. Then $Q$ is a normal subgroup of $G$ and $Q \leq K$. Moreover, by Proposition 1(i), $Q$ is prefactorized in $G$. Arguing as above we obtain $Q=1$. This contradicts the choice of $Q$. Thus we may suppose that $p$ is the largest prime dividing the order of $G$ and $G$ has a normal Sylow $p$-subgroup, $P$ say and $P K / K=N / K$.

Now we apply Lemma 4 to $G / K$ and analyze all the cases appearing there. Assume we are in the hypotheses of Lemma 4(ii). Then $G_{1} K / K=$ $G_{2} K / K=\ldots=G_{r} K / K=N / K$ for some $r<k$ and $\left(G_{r+1} G_{r+2} \ldots G_{k}\right) K / K$ is a complement of $N / K$ in $G / K$. Since $K \cap G_{i}=1$ for all $i=1,2, \ldots, k$, we have $\left|G_{i}\right|=p$ for $i=1,2, \ldots, r$ and $G_{j}$ is an abelian $p^{\prime}$-group for $j>r$. Further $P=G_{1} G_{2} \ldots G_{r}$ is the normal Sylow $p$-subgroup of $G$ and $G_{r+1} G_{r+2} \ldots G_{k}$ is a Hall $p^{\prime}$-subgroup of $G$. In particular, $P$ is a prefactorized subgroup of $G$. The above arguments imply that $G=C^{g} P=C P$. Hence the nilpotent residual $G^{\mathcal{N}}$ of $G$ is contained in $P$.

On the other hand, $P=G_{1} G_{2} \ldots G_{r}$ with $\left|G_{i}\right|=p$ for all $i=1,2, \ldots, r$. 
Assume that $G_{i}=G_{j}$ for $i \neq j \in\{1,2, \ldots, r\}$. Then $G$ can be regarded as a pairwise totally permutable product of less than $k$ factors. The choice of $G$ implies that there is a conjugate of $C$ which is prefactorized with respect to this new factorization. This clearly implies that this conjugate is actually prefactorized with respect to $G=G_{1} G_{2} \ldots G_{k}$. Hence we may assume that $G_{i} \neq G_{j}$ for $i \neq j \in\{1,2, \ldots, r\}$. This clearly implies that $\left[G_{i}, G_{j}\right]=1$ for all $i \neq j, i, j \in\{1,2, \ldots, r\}$, that is, $P$ is elementary abelian. Next we prove that $P=G_{1} \times G_{2} \times G_{3} \times \ldots \times G_{r}$. Assume that $G_{1} \cap\left(G_{2} G_{3} \ldots G_{r}\right) \neq 1$. Then $G_{1}$ is contained in $G_{2} G_{3} \ldots G_{r}$ and then $G=G_{2} G_{3} \ldots G_{k}$. By the choice of $G$, there exists a Carter subgroup $T$ of $G$ which is prefactorized with respect to this new factorization. This clearly implies that $T$ is prefactorized in $G=G_{1} G_{2} \ldots G_{k}$. Hence we have that $G_{1} \cap\left(G_{2} G_{3} \ldots G_{r}\right)=1$. Repeating the argument with the other factors, we have that $P$ is the direct product of $G_{1}, G_{2}, \ldots, G_{r}$. In particular, $G^{\mathcal{N}}$ is abelian and so it is complemented in $G$ by $C$ (see $[11, \mathrm{IV} ; 5.18]$ ). If $P=G^{\mathcal{N}}$, then $C$ would be a Hall $p^{\prime}$-subgroup of $G$ and, by Proposition 1(ii), $C$ would have a prefactorized conjugate, contrary to hypothesis. Hence $G^{\mathcal{N}}$ is a proper subgroup of $P$. By Theorem $2, G^{\mathcal{N}}=\left(G^{\mathcal{N}} \cap G_{1}\right)\left(G^{\mathcal{N}} \cap G_{2}\right) \ldots\left(G^{\mathcal{N}} \cap G_{r}\right)$. Since all the factors $G_{i}$ have order $p$, it follows that $G^{\mathcal{N}}$ is the product of all factors $G_{j}$ which are contained in $G^{\mathcal{N}}$. Without loss of generality, we may assume that $G^{\mathcal{N}}=G_{1} G_{2} \ldots G_{h}$ for some $h<r$. Let $T=\left(G_{h+1} G_{h+2} \ldots G_{r}\right)\left(G_{r+1} G_{r+2} \ldots G_{k}\right)$. Thus $G=G^{\mathcal{N}} T$ and $G^{\mathcal{N}} \cap T=1$. Furthermore $T$ is a Carter subgroup of $G$ which is clearly prefactorized, against supposition.

Now we assume $G / K$ satisfies Lemma $4(\mathrm{i})$. If $G_{1} K / K=G_{2} K / K=$ $\ldots G_{k} K / K=N / K$, then $G / K=N / K$ is abelian. Hence $M / K=1$, that is, $M=K$, contrary to assumption. Therefore there exists $i \in\{1,2, \ldots, k\}$, we suppose $i=1$, such that $G / K=G_{1} K / K$ and $G_{i} K / K=N / K$ for all $i \neq 1$. Since $K \cap G_{i}=1$ for all $i$, we have $\left|G_{i}\right|=p$ for all $i \neq 1$, $G=G_{1} K$ and $M=K\left(M \cap G_{1}\right)$. Thus $M \cap G_{1}$ is a $p^{\prime}$-group. Moreover $N=G_{2} G_{3} \ldots G_{k}\left(N \cap G_{1}\right)$. Now $\left(N \cap G_{1}\right) K / K$ is a subgroup of $N / K$ which is of order $p$. Hence either $\left(N \cap G_{1}\right) K / K=1$ or $N=\left(N \cap G_{1}\right) K$. If $\left(N \cap G_{1}\right) K / K=1$, then $N \cap G_{1} \leq K \cap G_{1}=1$. Therefore $N=G_{2} G_{3} \ldots G_{k}$ and $G=G_{1} N$. Furthermore $G / N$ is abelian. Thus $G_{1}$ is also abelian. But then $G / K$ is abelian and $M=K$, contrary to supposition. Consequently $N=\left(N \cap G_{1}\right) K$ and $\left|N \cap G_{1}\right|=p$. In particular, $N$ and $K$ are both $p$ groups. Now $G=N M=N K\left(M \cap G_{1}\right)=N\left(M \cap G_{1}\right)$. Thus $N$ is the Sylow $p$-subgroup of $G$ and $M \cap G_{1}$ is a Hall $p^{\prime}$-subgroup of $G$. Also $N \cap G_{1}$ is a Sylow $p$-subgroup of $G_{1}$, and $G_{1}=\left(N \cap G_{1}\right)\left(M \cap G_{1}\right)$. Arguing as in case (ii), 
we obtain that $N$ is elementary abelian. We prove that $N=\left(N \cap G_{1}\right) \times G_{2} \times$ $G_{3} \times \ldots \times G_{k}$. Suppose that $G_{2} \leq\left(N \cap G_{1}\right) G_{3} \ldots G_{k}$, then $G=G_{1} G_{3} \ldots G_{k}$ and, by the choice of $G$, there exists a Carter subgroup prefactorized with respect to $G=G_{1} G_{3} \ldots G_{k}$. Now an standard argument shows that this Carter subgroup is factorized with respect to $G=G_{1} G_{2} \ldots G_{k}$. This is a contradiction. If $N \cap G_{1} \leq G_{2} G_{3} \ldots G_{k}$, then $G=\left(M \cap G_{1}\right) G_{2} G_{3} \ldots G_{k}$. By the choice of $G$, there exists a Carter subgroup $T$ of $G$ such that $T=$ $\left(T \cap M \cap G_{1}\right)\left(T \cap G_{2}\right) \ldots\left(T \cap G_{k}\right) \leq\left(T \cap G_{1}\right)\left(T \cap G_{2}\right) \ldots\left(T \cap G_{k}\right) \leq T$, and $T$ is prefactorized, against supposition. Note that this argument could be used with every factor of the decomposition of $N$. Consequently $N=$ $\left(N \cap G_{1}\right) \times G_{2} \times G_{3} \times \ldots \times G_{k}$. In particular, $G^{\mathcal{N}} \leq N$ is abelian and so it is complemented in $G$ by $C$ (see $\left[11\right.$, IV; 5.18]). If $N=G^{\mathcal{N}}$, then $C$ would be a Hall $p^{\prime}$-subgroup of $G$ and, by Proposition 1(ii), $C$ would have a prefactorized conjugate, contrary to hypothesis. Hence $G^{\mathcal{N}}$ is a proper subgroup of $N$. By Theorem $2, G^{\mathcal{N}}=\left(G^{\mathcal{N}} \cap G_{1}\right)\left(G^{\mathcal{N}} \cap G_{2}\right) \ldots\left(G^{\mathcal{N}} \cap G_{k}\right)=\left(G^{\mathcal{N}} \cap N \cap G_{1}\right)\left(G^{\mathcal{N}} \cap\right.$ $\left.G_{2}\right) \ldots\left(G^{\mathcal{N}} \cap G_{k}\right)$. Assume first that $G^{\mathcal{N}} \cap G_{1}=1, G_{i} \leq G^{\mathcal{N}}$ for $i=1,2, \ldots, h$ and $G_{j} \cap G^{\mathcal{N}}=1$ for $i=h+1, \ldots, k$. Then $G=G^{\mathcal{N}}\left(G_{h+1} \ldots G_{k}\right)(N \cap$ $\left.G_{1}\right)\left(M \cap G_{1}\right)=\left(G_{2} \ldots G_{h}\right)\left(G_{h+1} \ldots G_{k}\right)\left(N \cap G_{1}\right)\left(M \cap G_{1}\right)$. Now let $T=$ $\left(G_{h+1} \ldots G_{k}\right)\left(N \cap G_{1}\right)\left(M \cap G_{1}\right)$. Arguing as in case (ii), $G^{\mathcal{N}} \cap T=1$ (recall that $N$ is a direct product of the subgroups $\left.\left(N \cap G_{1}\right), G_{2}, \ldots, G_{k}\right)$. Therefore $T$ is a Carter subgroup of $G$ and by construction it is prefactorized. On the other hand, if $N \cap G_{1} \leq G^{\mathcal{N}}$, then a similar argument yields $G^{\mathcal{N}}=$ $\left(N \cap G_{1}\right) G_{2} \ldots G_{h}$ and $G=G^{\mathcal{N}} T$ where $T=G_{h+1} \ldots G_{k}\left(M \cap G_{1}\right)$ is a Carter subgroup of $G$ and it is clearly prefactorized in $G$. We reach a contradiction in both cases.

Assume we are in case (iii) of Lemma 4 . Note that if $G_{j} K / K=1$ for $j \geq r+1$, then as $G_{j} \cap K=1$, we obtain $G_{j}=1$ for all $j \geq r+1$. Therefore we will be in case (i).

Finally if we are in the hypotheses of (iv) in Lemma 4 , we have $G / K=$ $G_{i} K / K$ for some $i$ and $G_{j} K / K=1$ for all $j \neq i$. As before, this means that $G_{j}=1$ for all $j \neq i$. Therefore $G=G_{i}$ and a Carter subgroup of $G$ is clearly prefactorized, the final contradiction.

Theorem 5. Let the group $G=G_{1} G_{2} \ldots G_{k}$ be the pairwise totally permutable product of the subgroups $G_{1}, G_{2}, \ldots, G_{k}$. Then $G$ has a prefactorized system normalizer.

Proof Denote by $r$ the nilpotent length of the soluble group $G$. Then $G \in$ $\mathcal{N}^{r}$, the class of soluble groups with nilpotent length at most $r$. If $r=2$, 
then by $[11, \mathrm{~V} ; 4.2]$, the system normalizers of $G$ coincide with the Carter subgroups of $G$. Therefore the conclusion follows by Theorem 4 . Thus we may assume that $r>2$. Set $G=H_{0}$ and denote by $H_{i}$ an $\mathcal{N}^{r-1-i} \mathcal{N}$-projector of $H_{i-1}$ for $i=1,2, \ldots, r-1$. By [11, V; 4.3(b)], $H_{r-1}$ is a system normalizer of $G$. We have the chain:

$$
1=H_{r} \leq H_{r-1} \leq \ldots \leq H_{1} \leq H_{0}=G
$$

Since the class $\mathcal{U}$ of all supersoluble groups is contained in $\mathcal{N}^{2} \subseteq \mathcal{N}^{r}$, we can apply Corollary 3 and Lemma 2(iv) to conclude that $H_{r-2}$ is factorized in $G$. Now $H_{r-1}$ is a Carter subgroup of $H_{r-2}$. Therefore, by Theorem 4 , there exists $h \in H_{r-2}$ such that $H_{r-1}^{h}$ is prefactorized in $H_{r-2}$. By Lemma 2(iv), $H_{r-1}^{h}$ is prefactorized in $G$. The proof of the theorem is now complete.

The following example shows that a Carter subgroup of a group need not be factorized.

Example 5 Consider the group

$$
G=\left\langle a, b, c \mid a^{3}=b^{7}=c^{7}=[b, c]=1, a^{2} b a=b^{2}, a^{2} c a=c^{2}\right\rangle
$$

$G$ is the totally permutable product of the subgroups $A=G$ and $B=\langle b\rangle$. Moreover the Carter subgroups of $G$ (which coincide with the system normalizers of $G$ ) are the conjugates of $\langle a\rangle$ which are not factorized in $G$.

In the final part of the section we study $\mathcal{F}$-projectors and $\mathcal{F}$-normalizers in mutually permutable products of two factors and obtain that they are not in general factorized although they are always prefactorized subgroups of the group.

Lemma 5. [7, Lemmas 1 and 2] Assume $G$ is the product of two mutually permutable subgroups $A$ and $B$. Then:

(i) If $N$ is a minimal normal subgroup of $G$, then $\{N \cap A, N \cap B\} \subseteq\{N, 1\}$.

(ii) If $N \leq A$ is a minimal normal subgroup of $G$ and $N \cap B=1$, then either $N \leq C_{G}(A)$ or $N \leq C_{G}(B)$; if furthermore $N$ is noncyclic, then $N \leq C_{G}(B)$.

(iii) If $N$ is a minimal normal subgroup of $G$ and $N \cap A=N \cap B=1$, then $|N|=p$, where $p$ is a prime, and either $N \leq C_{G}(A)$ or $N \leq C_{G}(B)$. 
Theorem 6. Let the group $G=A B$ be the mutually permutable product of the subgroups $A$ and $B$. If $\mathcal{F}$ is a saturated formation containing the class $\mathcal{U}$ of all supersoluble groups, then every sub-F-F-abnormal subgroup of $G$ is prefactorized.

Proof Assume the result is false and let $G$ be a counterexample of minimal order. Then there exists a sub- $\mathcal{F}$-abnormal subgroup $S$ of $G$ which is not prefactorized. Let us take $S$ of maximal order. If $S$ is not a maximal subgroup of $G$, then there exists $M$ an $\mathcal{F}$-abnormal maximal subgroup of $G$ with $S \leq M$ and $S$ is sub- $\mathcal{F}$-abnormal in $M$. By the choice of $S$, $M=(M \cap A)(M \cap B)$. Now the minimality of $G$ implies $S$ is prefactorized in $M$ and therefore in $G$ by Lemma 2(iv). Consequently, we may suppose that $S$ is an $\mathcal{F}$-abnormal maximal subgroup of $G$.

Then $G$ satisfies the following properties:

(i) $\operatorname{Core}_{G}(A \cap B)=1$.

Assume there exists a minimal normal subgroup $N$ of $G$ such that $N \leq A \cap B$. Suppose that $N \leq S$. Now $S / N$ is an $\mathcal{F}$-abnormal subgroup of $G / N$. By induction, $S / N$ is prefactorized in $G / N$. But $N$ is prefactorized in $G$. By Lemma 2(ii), $S$ is prefactorized, contrary to supposition. Therefore $G=S N, S \cap N=1, A=N(S \cap A)$ and $B=N(S \cap B)$. Consequently $G=N S=N(S \cap A)(S \cap B)$. Therefore $S=(S \cap A)(S \cap B)$, which contradicts the choice of $G$.

(ii) Every minimal normal subgroup of $G$ is contained in $S$.

Assume there exists a minimal normal subgroup $M$ of $G$ which is not contained in $S$. Then $G=S M$.

(a) Suppose that $M \cap A=M \cap B=1$. Then by Lemma 5(iii) $|M|=p, p$ a prime number. Therefore $G / C_{G}(M)$ is an $\mathcal{F}$-group and $G^{\mathcal{F}} \leq C_{G}(M)$. Applying [11, IV; $\left.1.17(\mathrm{~b})\right], S^{\mathcal{F}} \leq G^{\mathcal{F}}$ and so $S^{\mathcal{F}}$ is normal in $G$. If $S^{\mathcal{F}}=1$, then $S \in \mathcal{F}$. In this case, $G=$ $S M$ would be the totally permutable product of two $\mathcal{F}$-subgroups. Therefore, by [3, Lemma 4], $G \in \mathcal{F}$, contrary to the fact that $S$ is $\mathcal{F}$-abnormal in $G$. Hence $S^{\mathcal{F}} \neq 1$. On the other hand, $G / S^{\mathcal{F}}$ is the totally permutable product of $S / S^{\mathcal{F}}$ and $M S^{\mathcal{F}} / S^{\mathcal{F}}$ and both are $\mathcal{F}$-groups. A new application of [3, Lemma 4] yields $G / S^{\mathcal{F}}$ is an $\mathcal{F}$-group and so $G^{\mathcal{F}}=S^{\mathcal{F}}$. The minimality of $G$ implies that $S / S^{\mathcal{F}}$ 
is prefactorized in $G / S^{\mathcal{F}}$ and, by Theorem $2, S^{\mathcal{F}}$ is prefactorized in $G$. By Lemma 2(ii), $S$ is prefactorized in $G$, against supposition.

(b) Suppose that $M=(M \cap A)(M \cap B)$. We know by (i) that $M$ is not contained in $A \cap B$. Assume that $M \leq A$ and $M \cap B=$ 1. Now $A=M(S \cap A)$. We claim that $S \cap A \neq 1$. Suppose, arguing by contradiction, that $S \cap A=1$. Then $A=M, G=$ $A B=M B=M S$ and $A \cap B=M \cap B=1$. Let $X$ be a subgroup of $A$ of prime order. Then $X=X B \cap M$ is normalized by $B$. Consequently, $X$ is normal in $G$ and $X=M$, that is, $|M|=p$, where $p$ is a prime. Now consider the quotient group $G /$ Core $_{G}(S)=\left(\operatorname{MCore}_{G}(S) /\right.$ Core $\left._{G}(S)\right)\left(S /\right.$ Core $\left._{G}(S)\right)$. It is a primitive group and $\operatorname{MCore}_{G}(S) / \operatorname{Core}_{G}(S)$ has order $p$. Thus $G / \operatorname{Core}_{G}(S)$ is supersoluble, contradicting the fact that $S$ is $\mathcal{F}$ abnormal in $G$. Hence $S \cap A \neq 1$. On the other hand, applying Lemma 5(ii), either $M$ is cyclic of prime order or $M \leq C_{G}(B)$. We prove that $S \cap A$ is a maximal subgroup of $A$. If $M$ has prime order it is clear that $S \cap A$ is a maximal subgroup of $A$. Assume that $M \leq C_{G}(B)$. Then $M$ is a minimal normal subgroup of $A$ and $S \cap A$ is a proper subgroup of $A$ because $M$ is not contained in $S$. Thus $S \cap A$ is a maximal subgroup of $A$. Suppose that $A \cap B$ is not contained in $S \cap A$. Then $A=(S \cap A)(A \cap B)$, since $A \cap B$ is a permutable subgroup of $A$. Thus $G=(S \cap A)(A \cap B) B=$ $(S \cap A) B$ and $S=(S \cap A)(S \cap B)$, contrary to the choice of $S$. Consequently we may assume that $A \cap B$ is contained in $S$. On the other hand, by $[9,3.5], A \cap B$ is a subnormal subgroup of $G$. Therefore $M$ normalizes $A \cap B$ by [11, A; 14.3]. Then $T=(A \cap B)^{G}=(A \cap B)^{S} \leq S$. If $A \cap B=1$, then the product of $A$ and $B$ is totally permutable and $S$ is factorized by Theorem 3. This contradiction yields $A \cap B \neq 1$. Since $A \cap B$ is contained in $(T \cap A)(T \cap B)$, it follows that $(T \cap A)(T \cap B)$ is a nontrivial factorized normal subgroup of $G$ contained in $S$. By induction, $S /(T \cap A)(T \cap B)$ is prefactorized in $G /(T \cap A)(T \cap B)$. Applying Lemma 2(ii), $S$ is prefactorized. This contradiction proves (ii).

Let $N$ be a minimal normal subgroup of $G$. Then $N \leq S$. If $N=$ $(N \cap A)(N \cap B)$, the minimal choice of $G$ implies that $S / N$ is prefactorized in $G / N$. By lemma 2 (ii) the same is true for $S$. This contradiction yields $N \cap A=N \cap B=1$ for all minimal normal subgroups of $G$. This 
is to say that $\operatorname{Core}_{G}(A) \operatorname{Core}_{G}(B)=1$, which contradicts [8, Theorem $1])$.

Our last example shows that there exist mutually permutable products in which the $\mathcal{U}$-projectors $(\mathcal{U}$-normalizers) are not factorized.

Example 6 Consider $G \simeq \Sigma_{4}=\left[C_{2} \times C_{2}\right]\left[C_{3}\right] C_{2}$ the symmetric group of degree 4 . Then $G=A B$ is the mutually permutable product of $A$ and $B$ where $A$ denotes the alternating group of degree 4 and $B \simeq\left[C_{2} \times C_{2}\right] C_{2}$. Now consider $H \simeq\left[C_{3}\right] C_{2}$ a subgroup of $G$ isomorphic to the symmetric group of degree 3 . Then it is an $\mathcal{U}$-projector of $G$ (it is also an $\mathcal{U}$ normalizer of $G$ ) which is prefactorized in $G$ but not factorized.

We bring the paper to a close with the following.

\section{Open questions}

(a) Can Theorems 4 and 5 be extended to pairwise mutually permutable products?

(b) Is Theorem 6 true for mutually permutable products with more than two factors?

\section{Acknowledgements}

The first author has been supported by the grant MTM2010-19938-C03-01 from MICINN (Spain).

\section{References}

[1] B. Amberg, S. Franciosi, F. de Giovanni Products of groups Clarendon Press, Oxford, 1992

[2] A. Ballester-Bolinches, M. C. Pedraza-Aguilera and M. D. Pérez-Ramos, Totally and mutually permutable products of finite groups Groups St. Andrews 1997 in Bath I, 65-68. London Math. Soc. Lecture Note Ser. 260. Cambridge University Press, Cambridge, 1999. 
[3] A. Ballester-Bolinches, M. C. Pedraza-Aguilera and M. D. Pérez-Ramos, On finite products of totally permutable groups, Bull. Austral. Math. Soc., 53 (1996), 441-445.

[4] A. Ballester-Bolinches, M. C. Pedraza-Aguilera and M. D. Pérez-Ramos, Finite groups which are products of pairwise totally permutable subgroups, Proc. Edinb. Math. Soc., 41 (1998), 567-572.

[5] A. Ballester-Bolinches, J. C. Beidleman, H. Heineken and M.C. PedrazaAguilera. On pairwise mutually permutable products, Forum Math., 21 (2009), 1081-1090.

[6] A. Ballester-Bolinches, J. C. Beidleman, H. Heineken and M. C. Pedraza-Aguilera. Local classes and pairwise mutually permutable products of finite groups, Documenta Math. 15 (2010), 255-265.

[7] J. C. Beidleman and H. Heineken, Mutually permutable subgroups and group classes, Arch. Math., 85 (2005), 18-30.

[8] J. C. Beidleman and H. Heineken, Group classes and mutually permutable products, J. Algebra, 297 (2006), 409-416.

[9] A. Carocca, p-supersolvability of factorized groups, Hokkaido Math. J., 21(1992), 395-403.

[10] A. Carocca and R. Maier, Theorems of Kegel-Wielandt type, Groups St. Andrews 1997 in Bath I, 195-201. London Math. Soc. Lecture Note Ser. 260. Cambridge University Press, Cambridge, 1999.

[11] K. Doerk and T. Hawkes, Finite Soluble Groups, Walter De Gruyter, Berlin/New York, 1992.

[12] R. Maier and P. Schmid, The embedding of quasinormal subgroups in finite groups, Math. Z., 131 (1973), 269-272.

A. BALlester-Bolinches

Departament d'Àlgebra, Universitat de València

Dr. Moliner 50, 46100 Burjassot, València (Spain)

e-mail: Adolfo.Ballester@uv.es 


\section{J.C. BeIDLEMAN}

Department of Mathematics, University of Kentucky Lexington, Kentucky 40506-0027 U.S.A.

e-mail: clark@ms.uky.edu

H. HEINEKEN

Institut für Mathematik, Universität Würzburg

Am Hubland, 97074 Würzburg (Germany)

e-mail: heineken@mathematik.uni-wuerzburg.de

M.C. Pedraza-Aguilera

Instituto Universitario de Matemática Pura y Aplicada Universidad Politécnica de Valencia,

Camino de Vera, 46022, Valencia (Spain)

e-mail: mpedraza@mat.upv.es 